\title{
Translational drag coefficients of assemblies of spheres with higher-order hydrodynamic interactions ${ }^{\text {a) }}$
}

\author{
George D. J. Phillies \\ Department of Chemistry, The University of Michigan, Ann Arbor, Michigan 48105
}

(Received 4 June 1984; accepted 22 June 1984)

Calculations were made of the anisotropic translation drag coefficients of rigid assemblies of spheres in a variety of arrays, including lines, circular equilateral polygons, disks (polygons with a central sphere), and stacked squares. The objective was to compare results obtained using polynomial expansions for the two-body hydrodynamic interaction tensor truncated at the $(a / r)^{1}$, $(a / r)^{3},(a / r)^{5}$, and $(a / r)^{7}$ levels. Calculations were also made with the three- and four-body hydrodynamic interaction tensors recently obtained by Mazur and van Saarlos, thereby examining the relative importance of two-, three-, and four-body interactions. There are substantial cancellations between terms of order $(a / r)^{5}$ and $(a / r)^{7}$, and between the three- and fourbody hydrodynamic interactions. For the arrays studied here the Rotne-Prager [two-body, $(a / r)^{3}$ ] hydrodynamic interaction tensor gives results which are at least as satisfactory as the results obtained from the full Mazur-van Saarlos [four-body, $(a / r)^{7}$ ] interaction tensors.

\section{INTRODUCTION}

The hydrodynamic properties of biological macromolecules of irregular shape have long been calculated by approximate methods. As shown by Bloomfield, de la Torre, and collaborators, ${ }^{1-3}$ one useful approach is to treat the macromolecule as an assembly of $N$ small spheres. Calculations are based on algebraic or numerical solution of the hydrodynamic interaction equations for the $N$ spheres; these equations give $3 N \times 3 N$ dimensional matrices which connect the forces, flows, and torques on the spheres. From these matrices, one may calculate the anisotropic translational and rotational diffusion coefficients and the viscosity increment for the macromolecule of interest. More recently, Allison and McCammon ${ }^{4}$ have used Brownian dynamics to study the motion of a set of hydrodynamically interacting beads, finding complete agreement with the results of the matrix methods. The Brownian dynamics scheme promises, however, to be applicable to problems for which the matrix approach would be impractical.

A major variation in this series of calculations has been in the assumed form of the fundamental hydrodynamic interaction tensor. Calculations have been made with the Oseen tensor

$$
\mathrm{T}_{i j}=\frac{1}{6 \pi \eta a} \mid \delta_{i j}+\frac{1}{8 \pi \eta r_{i j}}\left[\mid+\hat{\mathbf{r}}_{i j} \hat{\mathbf{r}}_{i j}\right]\left(1-\delta_{i j}\right)
$$

in its full and spherically averaged forms, as well as with the modified Oseen tensor of Rotne and Prager ${ }^{5}$

$$
\begin{aligned}
\mathrm{T}_{i j} & =\frac{1}{6 \pi \eta a} \mid \delta_{i j}+\frac{1}{8 \pi \eta r_{i j}}\left(1-\delta_{i j}\right) \\
& \times\left[\left(I+\hat{r}_{i j} \hat{r}_{i j}\right)+\frac{a^{2}}{r_{i j}^{2}}\left(\frac{1}{3} \mid-\hat{\mathbf{r}}_{i j} \hat{\mathbf{r}}_{i j}\right)\right] .
\end{aligned}
$$

Here $\eta$ is the solvent viscosity, $a$ is the sphere radius, $\hat{\mathbf{r}}_{i j}$ and $r_{i j}$ are the unit vector and the distance between spheres $i$ and

\footnotetext{
'This work supported in part by the National Science Foundation under Grant CHE82-13941.
}

$j, \mathrm{I}$ is the identity tensor, $\delta_{i j}$ is the Kronecker delta $\left(\delta_{i j}=1, i=j\right.$, and $\left.\delta_{i j}=0, i \neq j\right)$, and ab denotes the outer (dyadic) product of the vectors $\mathbf{a}$ and $\mathbf{b}$.

It is well known that the Oseen and Rotne-Prager tensors are the lowest order terms in a power series (in $a / r$ ) expression for the exact hydrodynamic interaction tensors for two spheres. Felderhof ${ }^{6}$ has obtained a form for $T_{i j}$ which includes terms through order $(a / r)^{7}$. If one extends the power series to incorporate terms beyond $(a / r)^{3}$, interactions between trios and quartets of spheres become mentionable. This author ${ }^{7}$ obtained the lowest-order $\left[(a / r)^{4}\right]$ term linking three interacting spheres. Simultaneously, Mazur and van Saarloos $^{8}$ obtained all hydrodynamic interactions through order $(a / r)^{7}$, which requires taking into account forces linking three or four (but not five) particles. Much of this work was anticipated in a little-noticed paper by Kynch. ${ }^{9}$ Mazur and van Saarloos's work is further significant in that they went beyond the translation-translation couplings (in which a force exerted on the fluid by one particle leads to a force exerted by the fluid on a second particle). These workers also obtained the full set of translation-rotation, rotation-translation, and rotation-rotation couplings, in which the force or torque exerted on a fluid by one particle leads to a force or torque exerted on the fluid by a second particle.

It may rationally be suggested that the use of a low order (in $a / r$ ) approximation for $\mathrm{T}_{i j}$, in calculations on a set of nearly touching spheres, is inappropriate. The series expansion for $\mathrm{T}_{i j}$ includes high as well as low-order terms; the high-order terms will be most important for spheres which are nearly in contact. Calculation using different approximations for $T_{i j}$ may therefore obtain substantially different numerical results. On the other hand, while the higher-order forms for $T_{i j}$ have greater numerical accuracy than the Oseen and Rotne-Prager expressions, the computational effort needed to take account of three and four body interactions is far greater than the effort needed to consider two body interactions.

The objectives of this paper are: (i) to study the relative 
numerical consequences of using different approximations for $T$; and (ii) to study the relative importance of two, three, and four body hydrodynamic interactions. Numerical computations were made on lines, plane polygons, disks (a plane polygon with a central sphere), and stacked squares. The following section treats the general methods which are used here. Section III presents quantitative results for freely rotating spheres. A discussion closes the paper.

\section{GENERAL METHODS}

Our basic approach differs but little from the approaches used previously. ${ }^{1-3}$ In a suspension of freely rotating spheres, in which external forces $F_{j}$ and external torques $\mathbf{T}_{j}$ are applied to the individual spheres, the induced velocity of each sphere is given by

$$
\mathbf{v}_{i}=\sum_{j} \boldsymbol{\mu}_{i j}^{\mathrm{TT}} \cdot \mathbf{F}_{j}+\sum_{j} \boldsymbol{\mu}_{i j}^{\mathrm{TR}} \cdot \mathbf{T}_{j} .
$$

To obtain this equation it was assumed that inertial effects are negligible and that the external forces are independent of time. The hydrodynamic interaction tensors $\boldsymbol{\mu}_{i j}^{\mathrm{TT}}$ and $\boldsymbol{\mu}_{i j}^{\mathrm{TR}}$ give the translation-translation and translation-rotation coupling between $i$ and $j .(i=j$ is allowed in the sum.) As shown in Refs. 1-3, Eq. (1) may be used to calculate the translational and rotational diffusion coefficients of a body of irregular shape. At the level of the Rotne-Prager tensor, the applied and resultant forces act at the center of each sphere, so induced torques do not appear; this leads to complications for computations of rotational diffusion, which have been solved. ${ }^{2-4}$

Neglecting torques and inverting Eq. (3), it is found that

$$
\mathbf{F}_{j}=\sum\left[\boldsymbol{\mu}_{i j}^{\mathrm{TT}}\right]^{-1} \cdot \boldsymbol{v}_{i} .
$$

Equation (4) is a matrix equation. For an $N$ particle system, $\boldsymbol{\mu}_{i j}^{\mathrm{TT}}$ is an $N \times N$ matrix whose individual elements are the tensors ( $3 \times 3$ matrices) $\mathrm{T}_{i j}$. One may equivalently view $\mu_{i j}^{\mathrm{TT}}$ as a $3 N \times 3 N$ matrix whose first three lines refer to the $x, y$, and $z$ axes for the first particle, whose next three lines refer to the $x, y$, and $z$ axes for the second particle, and so on. The individual $3 \times 3$ element tensors $\mathrm{T}_{i j}$ which comprise $\boldsymbol{\mu}_{i j}^{\mathrm{TT}}$ are determined by the positions of all of the particles in the system; these tensors may be written as sums of two, three, four,...body terms, in which the $i-j$ coupling is modulated by each of the other particles in the suspension.

On requiring that all spheres move with unit velocity $u$ along one of the hydrodynamic axes of the assembly, Eq. (4) becomes

$$
\mathbf{f}=\sum_{j} \mathbf{F}_{j}=\operatorname{Tr}\left[\left[\boldsymbol{\mu}_{i j}^{\mathrm{TT}}\right]^{-1} \cdot \mathbf{u}\right] .
$$

$\operatorname{Tr}[$ ] denotes the trace operator. $f$ is the total force needed to produce unit velocity; the magnitude of $f$ is numerically equal to the translational drag coefficient for motion parallel to $u$. To calculate the anisotropic drag coefficients $f_{x}, f_{y}$, and $f_{z}$ of a sphere assembly, one only needs to obtain the individual $3 \times 3$ tensors $T_{i j}$, and perform conventional matrix operators.

What are suitable forms for the $T_{i j}$ ? The Oseen and Rotne-Prager approximations for the components of $T_{i j}$ are given in Eqs. (1) and (2). Mazur and van Saarlos have obtained the two-, three-, and four-particle hydrodynamic interaction tensors for groups of spheres which need not all have the same radius. These tensors differ from those given in Eqs. (1) and (2) in that T contains terms which depend on the coordinates of particles other than $i$ and $j$. Specifically, from Ref. 8 one may write

$$
\mathrm{T}_{i i}=\frac{1}{f_{0}}\left(1+\sum_{i \neq i} \mathbf{b}_{i l}+\sum_{k \neq i, l} \sum_{l \neq i, k} \mathbf{b}_{i l k}\right)
$$

while for $i \neq j$,

$$
\mathrm{T}_{i j}=\mathrm{T}_{i j}^{(2)}+\sum_{m} \mathrm{~T}_{i m j}^{(3)}+\sum_{m, n} \mathrm{~T}_{i m n j}^{(4)} .
$$

Denoting $f_{0}=6 \pi \eta a$, where $a$ is the radius of a sphere (all spheres are assumed in this paper to be the same size)

$$
\mathrm{b}_{i l}=-\frac{15}{4}\left(\frac{a}{r_{i l}}\right)^{4} \hat{r}_{i l} \hat{r}_{i l}+\frac{1}{16}\left(\frac{a}{r_{i l}}\right)^{6}\left[105 \hat{\mathbf{r}}_{i l} \hat{\mathbf{r}}_{i l}-171\right]
$$

and

$$
\begin{aligned}
\mathbf{b}_{i k l}= & \frac{75}{16} \frac{a^{7}}{r_{i k}^{2} r_{k l}^{3} r_{i l}^{2}}\left\{\left[1-3\left(\hat{\mathbf{r}}_{i k} \cdot \hat{\mathbf{r}}_{k l}\right)^{2}\right]\right. \\
& \times\left[1-3\left(\hat{\mathbf{r}}_{k l} \cdot \hat{\mathbf{r}}_{l i}\right)^{2}\right]+6\left(\hat{\mathbf{r}}_{i k} \cdot \hat{\mathbf{r}}_{k l}\right)^{2}\left(\hat{\mathbf{r}}_{k l} \cdot \hat{\mathbf{r}}_{l i}\right)^{2} \\
& \left.-6\left(\hat{\mathbf{r}}_{i k} \cdot \hat{\mathbf{r}}_{k l}\right)\left(\hat{\mathbf{r}}_{k l} \cdot \hat{\mathbf{r}}_{l i}\right)\left(\hat{\mathbf{r}}_{l i} \cdot \hat{\mathbf{r}}_{i k}\right)\right\} \hat{\mathbf{r}}_{i k} \hat{\mathbf{r}}_{l i} .
\end{aligned}
$$

For $i \neq j$ :

$$
\begin{aligned}
T_{i j}^{(2)}= & \frac{1}{f_{0}}\left\{\frac{3}{4} \frac{a}{r_{i j}}\left[1+\hat{\mathbf{r}}_{i j} \hat{\mathbf{r}}_{i j}\right]\right. \\
& \left.+\frac{1}{2}\left(\frac{a}{r_{i j}}\right)^{3}\left[1-3 \hat{\mathbf{r}}_{i j} \hat{\mathbf{r}}_{i j}\right]+\frac{74}{4}\left(\frac{a}{r_{i j}}\right)^{7} \hat{\mathbf{r}}_{i j} \hat{\mathbf{r}}_{i j}\right\}
\end{aligned}
$$

is the two-particle interaction tensor truncated at $(a / r)^{7}$. If $i$, $m$ and $j$ refer to distinct particles, $\mathbf{r}_{i m}$ being the vector from $i$ to $m$, and $\mathbf{r}=\mathbf{r}_{i m}, \mathbf{s}=\mathbf{r}_{m j}, r=\left|\mathbf{r}_{i m}\right|$, etc., one has

$$
\begin{aligned}
\mathrm{T}_{i m j}^{(3)}(r, s)= & -15 a^{4} /\left(8 r^{2} s^{2}\right)\left[1-3(\hat{\mathbf{r}} \cdot \hat{\mathbf{s}})^{2}\right] \hat{\mathbf{r}} \hat{\mathbf{s}}+3 a^{6} /\left(8 r^{2} s^{4}\right)\left[\left(1-5(\hat{\mathbf{r}} \cdot \hat{\mathbf{s}})^{2}\right) \hat{\mathbf{r}} \hat{\mathbf{s}}+2(\hat{\mathbf{r}} \cdot \hat{\mathbf{s}}) \hat{\mathbf{r}} \hat{\mathbf{r}}\right] \\
& +3 a^{6} /\left(8 r^{4} s^{2}\right)\left[\left(1-5(\hat{\mathbf{r}} \cdot \hat{\mathbf{s}})^{2}\right) \hat{\mathbf{r}} \hat{\mathbf{s}}+2(\hat{\mathbf{r}} \cdot \hat{\mathbf{s}}) \hat{\mathbf{s}} \hat{\mathbf{s}}\right]+a^{6} /\left(64 r^{3} s^{3}\right)\left\{\left[49-117(\hat{\mathbf{r}} \cdot \hat{\mathbf{s}})^{2}\right] 1-\left[93-315(\hat{\mathbf{r}} \cdot \hat{\mathbf{s}})^{2}\right]\right. \\
& \times(\hat{\mathbf{r}} \hat{\mathbf{r}}+\hat{\mathbf{s}} \hat{\mathbf{s}})+54(\hat{\mathbf{r}} \cdot \hat{\mathbf{s}}) \hat{\mathbf{s}} \mathbf{\hat { r }}+\left[729-1575(\hat{\mathbf{r}} \cdot \hat{\mathbf{s}})^{2}\right](\hat{\mathbf{r}} \cdot \hat{\mathbf{s}}) \hat{\mathbf{r}} \hat{\mathbf{s}} .
\end{aligned}
$$

If $i, m, n$, and $j$ refer to different particles, with $\mathbf{r}=\mathbf{r}_{i m}, \mathbf{s}=\mathbf{r}_{m n}$, and $\mathbf{t}=\mathbf{r}_{n j}$, one has

$$
\mathrm{T}_{i m n j}^{(4)}(r, s, t)=75 a^{7} /\left(16 r^{2} t^{3} s^{2}\right)\left\{\left[1-3(\hat{\mathbf{r}} \cdot \hat{\mathbf{t}})^{2}\right]\left[1-3(\hat{\mathbf{t}} \cdot \mathbf{s})^{2}\right]+6(\hat{\mathbf{r}} \cdot \hat{\mathbf{t}})^{2}(\hat{\mathbf{t}} \cdot \hat{\mathbf{s}})-6(\hat{\mathbf{r}} \cdot \hat{\mathbf{t}})(\hat{\mathbf{t}} \cdot \mathbf{s})(\hat{\mathbf{s}} \cdot \hat{\mathbf{r}})\right\} \hat{\mathbf{r}} \mathbf{s} .
$$

Physically, the tensor $\mathbf{b}_{i l}$ describes the retardation in the motion of a particle $i$ due to the presence of each of the other particles $l$. The tensor $\mathbf{b}_{i k l}$ describes the additional retardation of the motion of $i$ due to all additional pairs $k, l$ of 
particles in the system. The tensors $T_{i j}^{(2)}, T_{i m j}^{(3)}$, and $T_{i m n j}^{(4)}$ describe the motion of a particle at point $i$ due to a force applied to the fluid at point $j$. $T_{i j}^{(2)}$ gives the particle motion due directly to the force $\mathbf{F}_{j}$, while $T_{i m j}^{(3)}$ and $T_{i m n j}^{(4)}$ describe how the modification of fluid flow by the freely rotating and translating particles $m$ and $n$ modify the flow pattern at $i$. The modifications are linear, so that the effects of particles $m_{1}$, $m_{2}, \ldots, n_{1}, n_{2}, \ldots$ are simply linear. Felderhof gives a simple physical picture for these tensors, in which the Oseen tensor describes how an applied force sets up a flow in the fluid, other particles simply moving with the flow; the scattering of the nonuniform flow by other particles $m, n$,...leads to the higher order corrections in $b$ and $T$. As emphasized by Felderhof, the velocity of a sphere in a spatially nonuniform flow is not the same as the velocity which the fluid would have had, at the point occupied by the center of the sphere, if the sphere were not present. The tensors $b$ and $T$ give the velocity of a particle at $i$, not the fluid velocity induced at $\mathbf{r}_{i}$ by the same forces. This distinction has important physical consequences; in particular, $\nabla \cdot T_{i j} \neq 0$ if one goes beyond the Rotne-Prager approximation.

\section{NUMERICAL RESULTS FOR ROTATING SPHERES}

In this section, numerical results for assemblies containing as many as 16 spheres are discussed. The spheres are taken to be freely rotating, as required if Eqs. (8)-(12) are to be applicable. The assemblies which were studied include:

(i) linear arrays of up to 16 spheres;

(ii) equilateral circular polygons containing 3 to 16 spheres;

(iii) disks, a disk being an equilateral circular polygon with an additional sphere at the center of symmetry. As spheres cannot overlap, the polygons had to include more than five spheres;

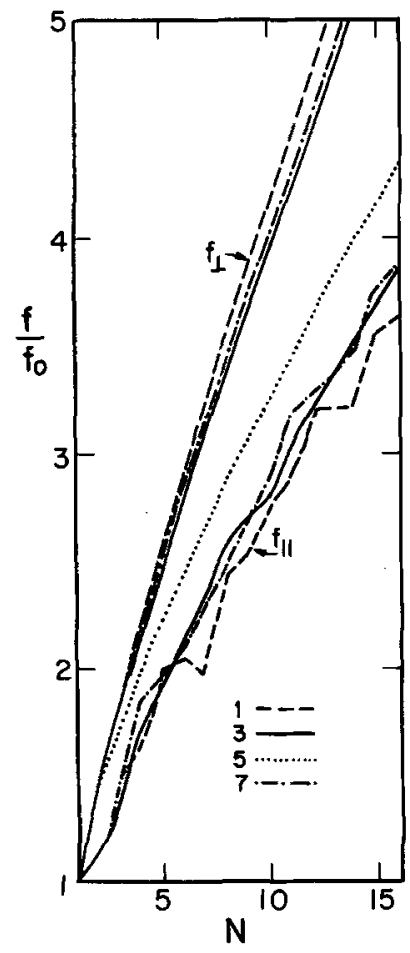

FIG. 1. Translational drag coefficients $f / f_{0}$ for linear arrays of $N$ spheres, using a two-body hydrodynamic interaction tensor truncated at $(a / r)^{n}$, for $n=1,3,5$, and $7 . f_{\|}$ and $f_{1}$ denote the drag coefficients for motions parallel and perpendicular to the $c_{\infty}$ axis of symmetry of the arrays.

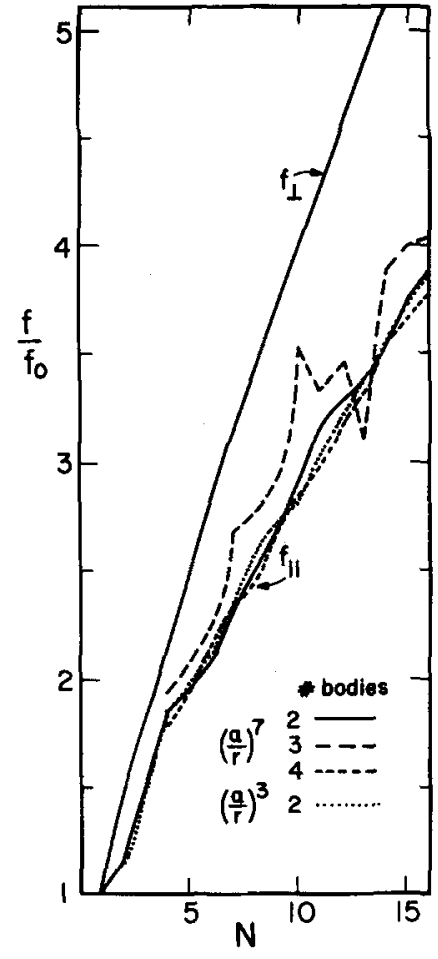

FIG. 2. Translational drag coefficients for linear arrays of $N$ spheres, using a hydrodynamic interaction tensor truncated at $(a / r)^{7}$ and including all hydrodynamic interaction terms involving $\boldsymbol{n}$ or fewer particles for $n=2,3$, or 4 . $f_{\|}$ and $f_{\perp}$ are as in Fig. 1. For linear arrays, $f_{1}$ is perceptibly independent of $n$. Also plotted is $f_{\|}$calculated from two-body hydrodynamics at the Rotne-Prager $\left[(a / r)^{3}\right]$ level; this relatively crude form for the hydrodynamics is seen to provide a reasonably good approximation for $f_{\|}$.

(iv) square lattices: sets for four spheres in a square, with further squares of four spheres stacked above the first square. The first two square lattices are the simple square and the cube.

In most cases, the spheres in an array were assumed to be touching, so that the distance $R$ between a sphere and its nearest neighbors was $2 a$. However, it is well known that the inversion of the matrix $\boldsymbol{\mu}_{i j}^{\mathrm{TT}}$ can be troubled by singularities;

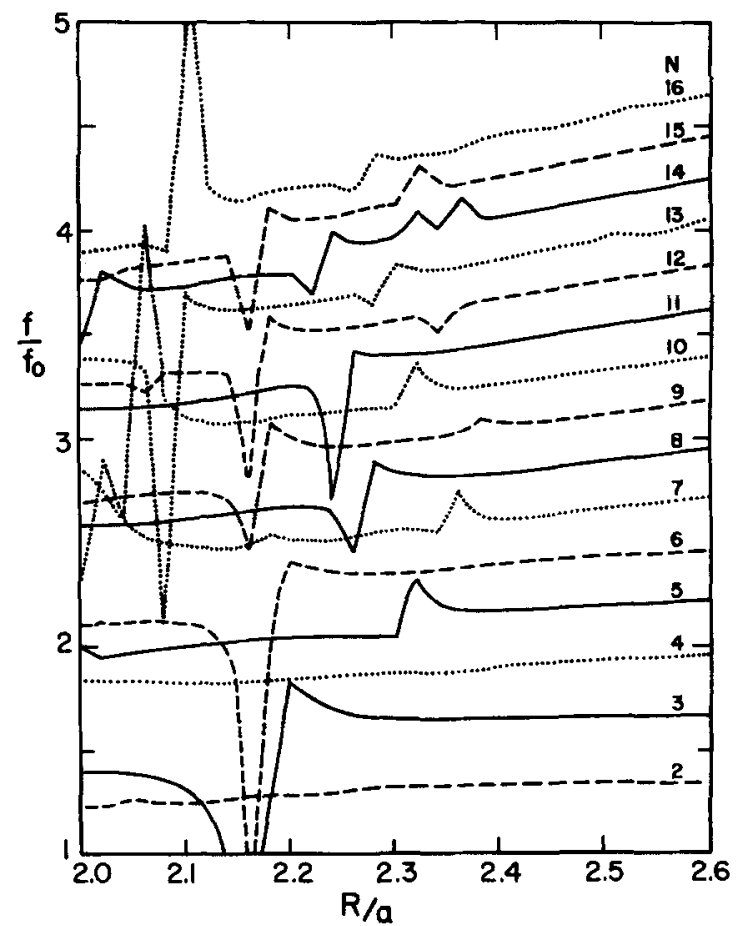

FIG. 3. Longitudinal drag coefficient of arrays of $N$ spheres of radius $a$, as a function of the center-to-center distance $R$ of the spheres. Calculations were made using two-body hydrodynamic interactions with truncation at $(a / r)^{7}$. Computations were made at intervals $\Delta R=0.02$; lines connect the computed points. 


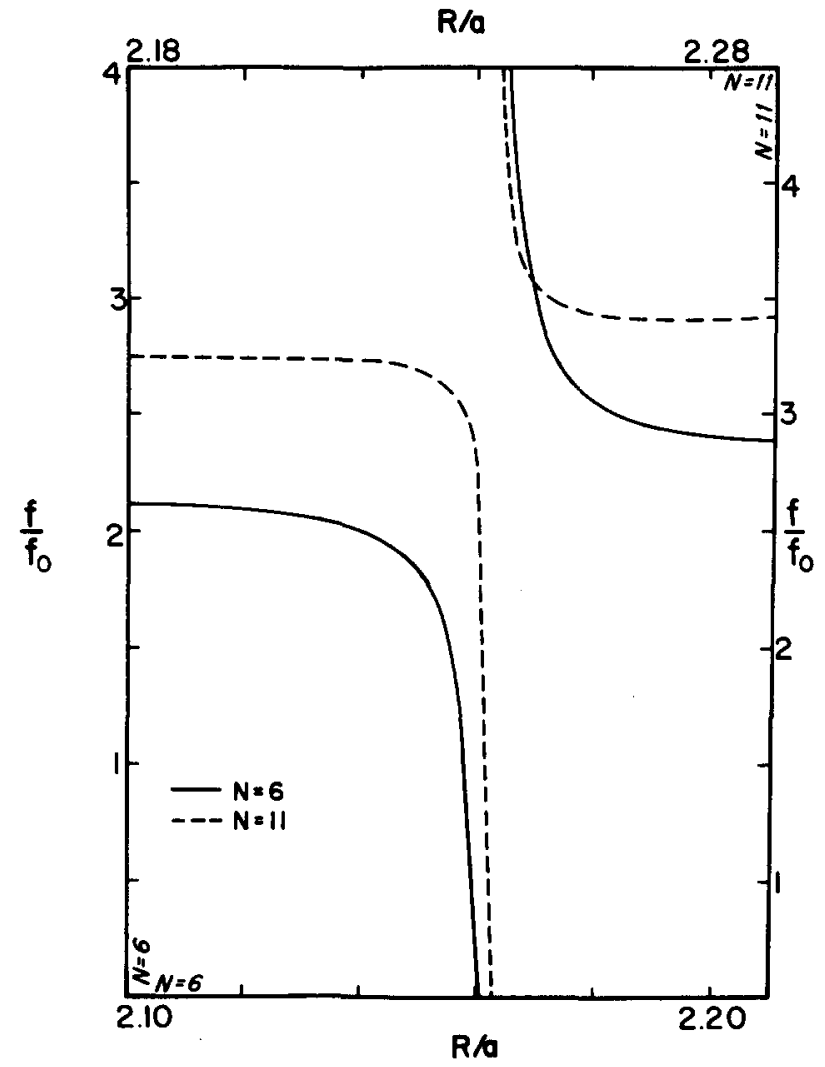

FIG. 4. Expanded sections of the $N=6$ and $N=11$ curves of Fig. 3, with $f_{\|} / f_{0}$ being evaluated at intervals $\Delta R=0.002 a$.

we therefore also studied the behavior of arrays of spheres as the distances between the spheres were gradually increased.

Figures 1-4 present results on linear arrays of identical spheres, using various approximations for the hydrodynamics. In Figs. 1 and 2, the nearest-neighbor distances are all $2 a$, so that each sphere touches its neighbors. In Figs. 3 and 4 , the dependence of the friction factor on the nearest neighbor distance $\boldsymbol{R}$ was examined. For a linear array, there are two anisotropic drag coefficients, the longitudinal drag coefficient $f_{\|}$(for motion parallel to the axis of symmetry) and the transverse drag coefficient $f_{1}$ (for motion perpendicular to the axis of symmetry).

Figure 1 shows $f / f_{0}$ as a function of the number of particles $N$. Calculations were made using the two-body hydrodynamic interaction tensors $T_{i j}^{(2)}$ and $b_{i l}$ of Eqs. (8) and (10), with truncation of the power series at several levels, including the Oseen $\left[(a / r)^{1}\right]$, Rotne-Prager $\left[(a / r)^{3}\right],(a / r)^{5}$, and Mazur-van Saarlos $\left[(a / r)^{7}\right]$ levels. These computations are indicated as $n=1,3,5$, and 7 , respectively. For transverse motion, all four approximations to $T_{i j}$ give nearly the same numbers. For $f_{\|}$, the results are somewhat more complex. If the Oseen tensor is used for $T_{i j}$, nearby singularities in $\left[\boldsymbol{\mu}_{i j}^{\mathrm{TT}}\right]^{-1}$ lead to an irregular and unphysical dependence of $f_{\|} / f_{0}$ on $N .^{10}$ The Rotne-Prager and $(a / r)^{7}$ approximation lead to very similar numbers for $f_{\|} / f_{0}$, but the $(a / r)^{5}$ approximation gives substantially larger values for $f_{\|} / f_{0}$ than do the other approximations.

Computations were also made, with truncation at the $(a / r)^{7}$ level, after including three-body interactions, and after including both three- and four-body interactions. A com- parison of the importance of two-, three-, and four-body interactions is shown in Fig. 2. The transverse drag coefficient is not significantly affected by the addition of three- and four-body terms. As was also seen in Fig. 1, the longitudinal drag coefficient is substantially more sensitive to singularities in the matrix inversion than is the transverse drag coefficient. Incorporation of three-body terms leads to an $f_{\|} / f_{0}$ with an irregular dependence on $\boldsymbol{N}$. These irregularities are largely suppressed when the four-body terms are added to the two- and three-body terms. Use of a hydrodynamic interaction tensor which is complete through order $(a / r)^{7}$ (which includes forces between as many as four bodies) results in an $f_{\|} / f_{0}$ which is not much different than the $f_{\|} / f_{0}$ obtained from the Rotne-Prager tensor. For linear arrays, including both the three and four particle interactions thus does not appear to have a substantial effect on $f$. The irregularities seen in the three-particle calculation may be due to a lack of symmetry in the way the calculation was performed. Many three-body terms are simply four-body terms in which the first and last bodies in a sequence are taken to be the same.

Figures 1 and 2 indicate that the longitudinal drag coefficient does not always show a smooth dependence on $N$. If the irregularities were simply due to a lack of complete convergence of the power series expansion for $T$, so that the short-range approximation for $T$ was not sufficiently accurate, $f_{\|} / f_{0}$ might be better behaved if the spacing between adjacent spheres were increased. To test this hypothesis, $f_{\|} / f_{0}$ for linear arrays of spheres was recalculated, using Eq. (10) for $T_{i j}$, as the distance $R$ between adjoining spheres was increased from $2.0 a$ to 2.6a. Results of this calculation are shown in Fig. 3. Computations were made at intervals of $0.02 a$; the indicated lines connect points of fixed $N$. In regions in which $f_{\|}$is changing rapidly, the lines may not be good approximations to the actual analytic dependence of $f$ on $R$. For $R>2.4 a, f_{\|} / f_{0}$ depends gently on $R$, as would be expected for a physical system. For $R<2.4 a, f_{\|} / f_{0}$ can depend markedly on $R$. It is not expected that the real bodies modeled by the sphere assemblies would show this behavior experimentally. For $R>2.4 a$, these anomalies are weak or

TABLE I. Drag coefficients of $N$-particle ring assemblies with order $(a / r)^{7}$ $m$-particle hydrodynamics.

\begin{tabular}{|c|c|c|c|c|c|c|c|}
\hline \multirow[b]{2}{*}{$N$} & \multirow{2}{*}{$\boldsymbol{M}$} & \multicolumn{3}{|c|}{ In plane } & \multicolumn{3}{|c|}{ Out of plane } \\
\hline & & 2 & 3 & 4 & 2 & 3 & 4 \\
\hline 4 & & 1.963 & 1.752 & 1.760 & 1.881 & 1.863 & 1.863 \\
\hline 5 & & 1.944 & 1.867 & 1.931 & 2.143 & 2.125 & 2.125 \\
\hline 6 & & 2.062 & 2.028 & 2.089 & 2.407 & 2.394 & 2.394 \\
\hline 7 & & 2.244 & 2.218 & 2.255 & 2.669 & 2.664 & 2.664 \\
\hline 8 & & 2.431 & 2.418 & 2.426 & 2.927 & 2.930 & 2.930 \\
\hline 9 & & 2.619 & 2.623 & 2.602 & 3.182 & 3.194 & 3.194 \\
\hline 10 & & 2.807 & 2.828 & 2.780 & 3.433 & 3.453 & 3.453 \\
\hline 11 & & 2.993 & 3.032 & 2.962 & 3.681 & 3.709 & 3.709 \\
\hline 12 & & 3.177 & 3.234 & 3.140 & 3.926 & 3.960 & 3.960 \\
\hline 13 & & 3.360 & 3.433 & 3.320 & 4.168 & 4.209 & 4.209 \\
\hline 14 & & 3.541 & 3.630 & 3.500 & 4.408 & 4.455 & 4.455 \\
\hline 15 & & 3.721 & 3.824 & 3.678 & 4.644 & 4.697 & 4.697 \\
\hline 16 & & 3.900 & 4.016 & 3.857 & 4.879 & 4.937 & 4.937 \\
\hline
\end{tabular}


absent. Physical values of $f_{\|}$for $R<2.4 a$ may apparently be obtained by extrapolation from larger $R$.

As is apparent in the $N=6$ curve, $f_{\|}$may be essentially normal when the spheres touch and then become anomalous as the spheres move apart. The anomalies in $f_{\|}$are therefore not due simply to inaccuracies in the interaction tensors [Eqs. (8) $-(12)]$ at short distances. If one were to calculate the drag coefficients of a single assembly with a single $R$, the presence of an anomaly might not be obvious. By repeating a computation while using a series of slightly different sphere spacings, resonance-like features in $f$ may readily be identified. It is interesting to note that if a value of $R$ leads to an anomalous curve for $f_{\|}$for some value of $N$, then the behavior of $f_{\|}$for $N+3, N+6$,...is also anomalous. For example, near $R=2.16 a$, anomalies are seen for $N=3,6,9,12$, and 15.

Figure 4 shows a calculation of $f_{\|} / f_{0}$ for $N=6$ and $N=11$, with calculations being made at intervals $\Delta R=0.002 a$. Over a narrow range of vaues of $R$, the anomalies can be rather deeper than is suggested by Fig. 3. As the anomalies are primarily of mathematical interest, we did not expend the large amounts of computer time needed to generate, for all of Fig. 3, curves as accurate as those shown in Fig. 4.

Computations were also made on circular equilateral polygons, with or without an additional sphere at the origin. Polygons were previously studied by Paul and $\mathrm{Mazo}^{11}$ at the Oseen tensor level. Figure 5 presents the drag coefficients $f_{o p}$ (for motion perpendicular to the plane of the polygon) and $f_{i p}$ (for motion in the plane of the polygon) for plane

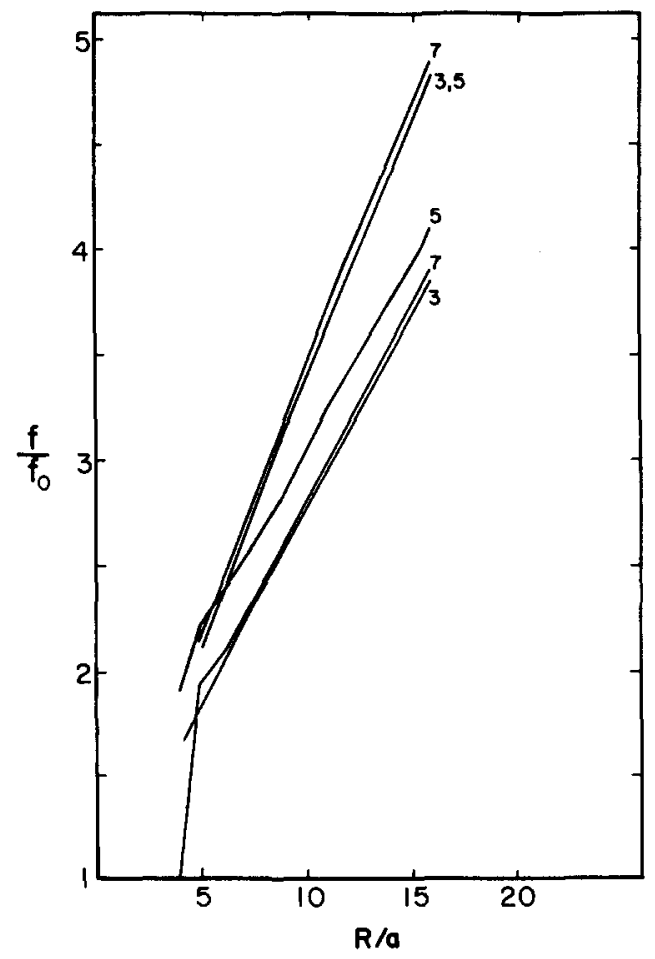

FIG. 5. In- and out-of-plane drag coefficients for equilateral circular polygons of $N$ spheres, computed with two-body hydrodynamic interactions and truncation of the power series at $(a / r)^{n}$ for $n=3,5$, and 7 . Note that the changes from $n=3$ to $n=5$ and from $n=5$ to $n=7$ have substantial but cancelling effects on the in-plane drag coefficient (lower lines). polygons of $N$ spheres. Separate computations were made with $\mathrm{T}_{i j}$ truncated at the $n=3,5$, and 7 levels. For $f_{o p}$, $n=3$ and 5 are essentially indistinguishable, while $n=7$ gives perhaps $2 \%$ larger values for $f$. For $f_{i p}$, results with the Rotne-Prager and Mazur-van Saarlos forms are very similar, though truncation at the $(a / r)^{5}$ level gives rise to a significantly bigger $f_{i p}$. For $N>4$, there is no indication of the singularities which were observed in calculations on the linear arrays. A square is, of course, a pair of parallel lines; results for squares do indicate singularities as treated below.

By placing a sphere at the center of a polygon, one can study hydrodynamic shielding. It is well known that nearby non-point-like particles can modify fluid flow around a given particle, so that a particle in the middle of an assembly will not be greatly affected by an external flow field. Polygons containing 6 to 15 spheres were modified by placing an additional sphere at the origin: the drag coefficients of the disklike arrays were then recalculated. The extent of screening is given in Fig. 6, which plots the increase in $f_{i p}$ and $f_{o p}$ when a sphere is added to an $N$-sphere polygon. The additional sphere makes only a very small change, typically less than $0.02 f_{0}$, in either $f_{i p}$ or $f_{o p}$. This confirms the importance of hydrodynamic screening for reducing the hydrodynamic importance of interior spheres, even when the distance between the ring and its central sphere becomes fairly large (as much as several sphere radii).

Figures 7 and 8 present the drag coefficients for square arrays of spheres containing 4, 8,12, or 16 spheres, the independent variable being the distance $R$ between the spheres. Here $f_{i p}$ refers to motion in the plane of a given square, while $f_{o p}$ refers to motion perpendicular to the plane of symmetry of a single square. For the cube these two drag coefficients are equal. Computations were made with two body hydrodynamics at the Rotne-Prager and $(a / r)^{7}$ orders of precision and with four-body hydrodynamics at the $(a / r)^{7}$ level. For the square these three approximations to $T$ give virtually identical values for $f$. For larger sphere assemblies, there are discrepancies. In the figures, the dashed halftone lines give results from the Rotne-Prager tensor. In all cases $f$ shows a smooth dependence on $R / a$, with no indication of singulari-

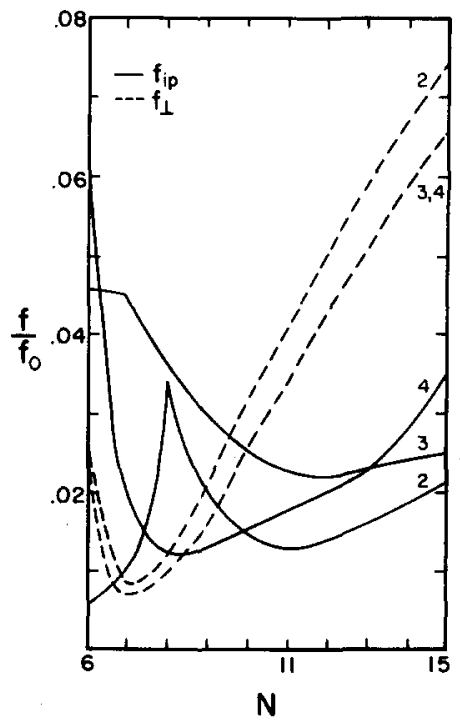

FIG. 6. The fractional change $f /$ $f_{0}$ in the drag coefficients of an $N$ sphere ring polygon occasioned by adding to the assembly a sphere placed at the origin. Computations were made at the $(a / r)^{7}$ level using two-, three-, and fourbody forces. A ring polygon almost completely screens a sphere at its center from the surrounding fluid flow, even when the polygon radius is several times the radius of the spheres. 


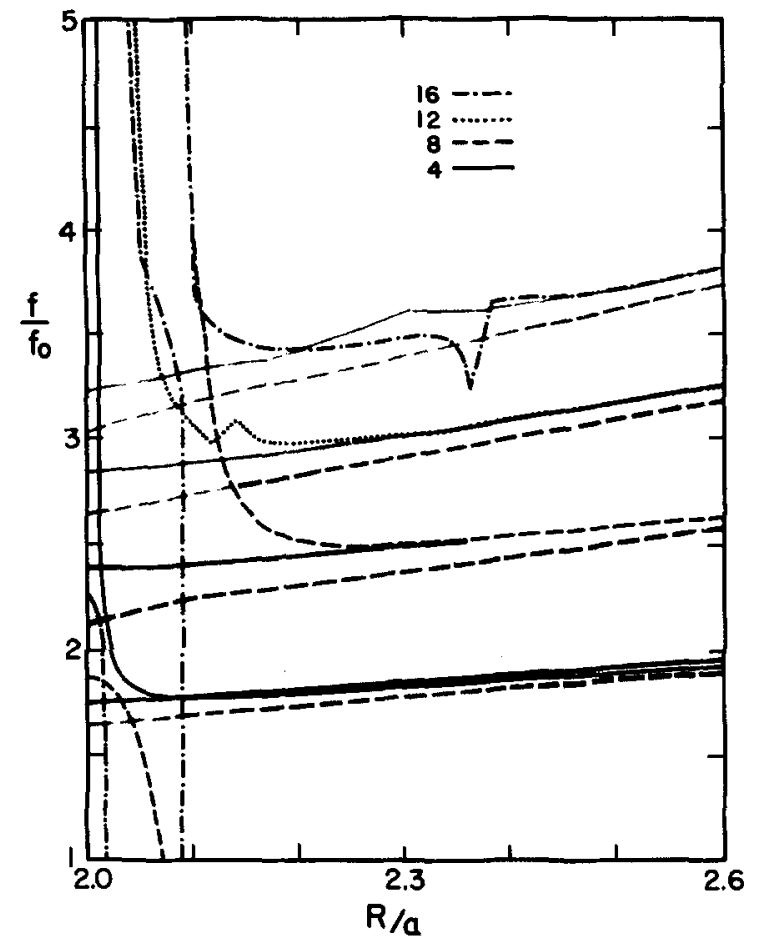

FIG. 7. Plot showing the in-plane drag coefficient of $n(n=1,2,3$, or 4) stacked squares as a function of the nearest-neighbor center-to-center distance $R$, in units of the sphere radius $a$. The plane is the plane formed by the four spheres of a single square. Computations were made with two-body ( $a$ ) $r)^{7}$ interactions (black lines), the full $n$-particle $(a / r)^{7}$ interaction tensors (solid gray lines), and the Rotne-Prager tensor (dashed gray lines).

ties. If one extends $T_{i j}$ to include pairwise interactions at the $(a / r)^{7}$ level, one finds the results indicated by the black lines; there are strong singularities in both drag coefficients, ex-

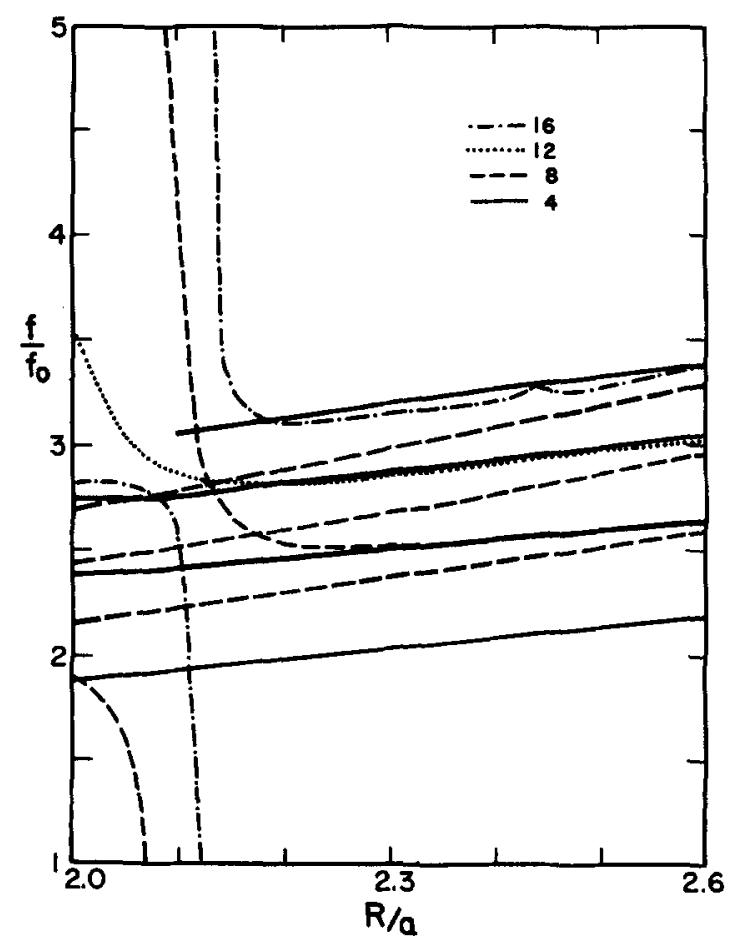

FIG. 8. The out-of-plane drag coefficients of $n(n=1,2,3$, or 4) stacked squares as a function of the nearest-neighbor center-to-center distance $R / a$. Other details are as in Fig. 7. tending out to substantial $(R>2.3 a)$ sphere separations. Almost all of these singularities are eliminated if three- and four-body interactions are taken into account, though the $f_{\text {op }}$ of the 16-sphere assembly is still strongly anomalous when the spheres are in contact with each other.

\section{DIscussion}

We have here presented extensive numerical computations of the drag coefficients of symmetric arrays of spheres. Contrasts have been shown between different possible levels of truncation of the power series expansions for the twobody hydrodynamic interaction tensor. The consequences of including three- and four-body hydrodynamic interactions were also studied. The magnitude of hydrodynamic screening effects were obtained by comparing drag coefficients of rings and disk-like arrays.

Our major result is that there are substantial fortuitious cancellations between terms of different order in $(a / r)$, and terms involving hydrodynamic interactions between various numbers of bodies. For most of the arrays studied here the Rotne-Prager two-body hydrodynamic interaction tensor gives results which are within a few percent of the results obtained using $(a / r)^{7}$ hydrodynamics, either at the two- or four-body level. For stacked arrays of squares, expressions for $T_{i j}$ more sophisticated than the Rotne-Prager often give far less plausible results for $f / f_{0}$. The treatment of an irregular body as an assembly of freely rotating spheres is at best a good approximation. In calculations of $f / f_{0}$, it would therefore appear the Rotne-Prager tensor is to be preferred to more sophisticated expressions for $T_{i j}$.

Instances were found in which $f / f_{0}$ shows anomalous behavior. In these cases the drag coefficients depend strongly but not monotonically on the sphere spacing $R$. These anomalies are greatly reduced if four as well as three-body interactions are taken into account, and are suppressed if the distance between neighboring spheres is increased. Thus, by testing the dependence of $f$ upon $R$, one may recognize the presence of singularities in $\mu^{-1}$. At larger $R(R>2.4 a), f / f_{0}$ only depends weakly on $R$. It may therefore be possible to calculate $f / f_{0}$ for relatively large values $R$, and then extrapolate inwards to smaller values of $R$. (The inversion of a matrix with near-zero eigenvalues can lead to difficulties when finite-precision arithmetic is performed. Some of our anomalous or normal results may therefore change if calculations are repeated at a different level of precision.)

There exist problems of theoretical interest in which the above conclusions do not apply. First, this paper only considers translational motion. For problems in which molecular rotation is important, such as the calculation of rotational diffusion coefficients, a separate analysis is necessary. In much previous work, expressions for $\mu^{\mathrm{TR}}$ and $\mu^{\mathrm{RR}}$ were not available; to express the effect of the torque, applied to the fluid by a given body, on the rotation of a second body, it was necessary to model each body as an array of spheres. By using the translation-rotation and rotation-rotation tensors of Mazur and van Saarlos, ${ }^{8}$ the rotational coupling of two spheres may be described.

Second, this paper has assumed that the spheres were freely rotating. If the spheres had fixed orientations, the 
higher-order approximations for $b_{i j}$ and $T_{i j}$ would be modified. With these modifications, there is no guarantee that the Rotne-Prager tensor is still a good approximation for T. Fixing the orientation of the spheres of an assembly requires that the assembly be able to transmit nonhydrodynamic (mechanical) stresses, which stresses would in general affect the effective drag coefficients of the assembly.

Third, these results do not apply if one is interested in the interactions between several independently moving bodies. As may be shown by direct analytic computation the translational diffusion coefficients for a set of interacting Brownian particles may (for short-range direct interactions) be given a virial-like expansion in the concentration $c .{ }^{12}$ The $c^{1}$ term depends only on pair interactions. The $c^{2}$ term is modified by the three-body interactions $\mathrm{b}_{i k l}$ and $\mathrm{T}_{i m j}$, but there cannot be a cancellation of these interactions by the
$\mathrm{T}_{i m n j}$ tensor, because $\mathrm{T}_{i m n j}$ first appears in the calculation at the $c^{3}$ level. The internal modes of polymer molecules might show similar behavior.

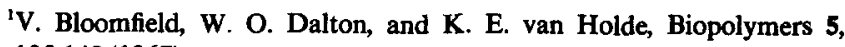
135,149 (1967).

${ }^{2} \mathrm{~J}$. G. de la Torre and V. A. Bloomfield, Biopolymers 17, 1605 (1978).

${ }^{3}$ J. M. G. Bernal and J. G. de la Torre, Biopolymers 20, 129 (1981).

${ }^{4} S$. A. Allison and J. A. McCammon, Biopolymers 23, 167 (1981).

${ }^{5}$ J. Rotne and S. Prager, J. Chem. Phys. 50, 4831 (1969).

${ }^{6}$ B. U. Felderhof, Physica A 89, 373 (1977).

${ }^{7}$ G. D. J. Phillies, J. Chem. Phys. 77, 2623 (1982).

${ }^{8}$ P. Mazur and W. van Saarlos, Physica A 115, 21 (1982).

${ }^{9}$ G. J. Kynch, J. Fluid Mech. 5, 193 (1959).

${ }^{10}$ R. W. Zwanzig, J. Kieffer, and G. H. Weiss, Proc. Natl. Acad. Sci. U.S.A. 60, 381 (1968).

"E. Paul and R. M. Mazo, J. Chem. Phys. 51, 1102 (1969).

${ }^{12}$ G. D. J. Phillies, J. Chem. Phys. 77, 2623 (1982). 